\title{
Analyzing Performance of Separating Algorithm on Responsive signals of the Transponders
}

\author{
Gong Fengxun,", Zhang Zhongyong ${ }^{2}$ and Ma Yanqiu ${ }^{2}$ \\ ${ }^{I}$ College of Electronic and Information Engineering Civil Aviation University of China, Tianjin, China \\ ${ }^{2}$ Air Traffic Management Bureau. CAAC, Beijing 100022, China
}

\begin{abstract}
Multiple 1090ES responsive signals may be overlapped in the receiver systems on the ground. At the same time, 1090ES respond signals may be interfered by A/C replies with the same carrier frequency. To address these problems, firstly, principle component analysis (PCA) is employed to pre-whiten the observed signals in order to reduce the relevance between signals. Then extended projection algorithm (EPA) is applied to separate 1090ES respond signals. Effect of the separated signal is verified by simulation. The performance of this algorithm is also analyzed in several aspects. Experiments results show that this algorithm not only can successfully in separating multiple 1090ES responsive signal effectively but also has both higher precision and strong ability of stability.
\end{abstract}

Keywords: Signals separation, Extended projection algorithm, Principle component analysis, Singular value decomposition

\section{INTRODUCTION}

Because airports are becoming more and more large and busy, and the airport scene motion planning and collision detection become very important. ICAO has focused on the research of Advanced Surface Movement Guidance and Control System (A-SMGCS), which represents the trend of future civil air traffic control in recent years. Responsive signals recognition and estimation are the core technology of surface location in the A-SMGCS. The responsive signals can be used for MLAT (Multilateration) and ADS-B systems in targets location in airports. 1090ES is the data link system recommended by ICAO using in commercial air transport. There are a lot of successful applications with responsive signals on surface positioning in hub airport in the world, such as Heathrow, Frankfurt, Chicago, etc. In 2007, Civil Aviation of China started the validation project of ADS-B in China by using ADS-B transceivers and other equipments produced by SENSIS company [1]. When aircrafts arrival to the airport, their position must be reported to controller. The situation must be bringing out that several 1090ES and/or ADS-B signals arrival ground receivers simultaneously. And then the 1090ES and/or ADS-B signals may be interfered by $\mathrm{A} / \mathrm{C}$ replies with the same carrier frequency. In [2], the authors achieve the separation of the two overlapping secondary radar signals using the Projection Algorithm (PA). However, the PA is only suitable for the case that the time delay offset between the two secondary radar signals is large enough and that the signal to noise ratio is high enough. In [3], the authors achieve the separation of radar signals with Multishift Zero-Constant Modulus Algorithm (MS-ZCMA).
This algorithm is very complex. The complex computation of MS-ZCMA will increase hardware requirements, because $1090 \mathrm{ES}$ and/or ADS-B signals need real-time processing. In [4], the Manchester Decoding Algorithm (MDA) is used to separate overlapped secondary radar signals. The MDA algorithm is only applicable to highly overlapped signals. When the time delay offset is greater than $40 \mu \mathrm{s}$, the bit error rate of the separated signals is very high. In this paper, the extended projection algorithm (EPA) is applied to separate multiple $1090 \mathrm{ES}$ and/or ADS-B signals overlapped with A/C responsive signals to find better results.

\section{EXPECTION SINGNAL MODEL ANALYSIS}

As a typical application in airports, the ADS-B signals model based on 1090 data link must be constructed and analyzed in here. According to the basic structure of ADS-B messages, the 1090 responsive signal contains 112 binary symbols $b_{n}$ (it is called 1090ES). The bits are encoded in "Manchester encoding" scheme, where $b_{n}=0$ is encoded as $b_{n}=[0,1]$ and $b_{n}=1$ as $b_{n}=[1,0]$. The transmitted bit stream b is a packet consisting of a preamble $\mathrm{p}=[1,0,1,0,0,0,0,1$, $0,1,0,0,0,0,0,0]$ which is followed by the encoded data bits. The preamble is aimed to detect the start of a reply from targets and determine whether the signal is 1090ES. The total length of $\mathrm{b}$ is 240 , corresponding to $120 \mu \mathrm{s}$, i.e., $b=\left[p, b_{1}, b_{2}, \cdots, b_{112}\right]$. And then the 1090ES signal can be described as follow:

$b(t)=\sum_{n=0}^{L} b[n] q(t-n T)$ 
Where $\mathrm{L}=239, \mathrm{~b}[\mathrm{n}]$ is the $\mathrm{n}$-th entry of $\mathrm{b}$, and $\mathrm{q}(\mathrm{t})$ is a rectangular pulse of width $\mathrm{T}=0.5 \mu \mathrm{s}$. Then the $\mathrm{b}(\mathrm{t})$ signal can be sampled with the frequency of $f$, and then it is represented as follow:

$s[n]=b\left[n T_{s}\right]$

where $T_{s}=1 / f_{s}$ is the sampling period.

Considering the reception of $\mathrm{d}$ independent source signals on an m-element Colinear-Coaxial-Antenna, and then assuming there is no multipath in signals transmission. The baseband signals $x_{i}[n]$ with $i \in[1, \cdots, m]$ are stacked in vectors $\mathrm{x}[\mathrm{n}]$ ( size $\mathrm{m})$. After collecting $\mathrm{K}$ samples, the observation matrix model is:

$X=M S+N$

Where, $X=[x[1], \cdots x[K]]$ is the $m \times K$ received signal matrix. $S=[s[1], \cdots, s[K]]$ is the $d \times K$ source matrix, in which $\mathrm{s}[n]=\left[s_{1}[n], \cdots, s_{d}[n]\right]^{T}$ is a stacking of the d source signals. $\mathrm{N}$ is the $m \times K$ noise matrix. $\mathrm{M}$ is the $m \times d$ matrix that contains the array signatures and the complex gains of the sources.

\section{ANALYSIS OF THE ALGORITHM PRINCIPLE}

\subsection{Principle Component Analysis}

There are mainly two aspects in PCA analysis: prewhitening procedure and dimension reduction procedure.

The purpose of the pre-whitening procedure is to separate the 1090ES signals irrelevantly [5]. And the purpose of dimension reduction is to reduce the received signal dimension $\mathrm{m}$ to $\mathrm{d}$ in order to reduce computational complexity. Firstly, using received signal matrix $X$ minus its mean $\mathrm{E}\{\mathrm{X}\}$, that is $\tilde{X}=X-E\{X\}$, and hypothesis $E\{\tilde{X}\}=0$. Secondly, the covariance matrix of $\tilde{X}$ can be obtained, and then the $C=E\left\{X X^{T}\right\}$ result is get. At last, the eigenvalue decomposition[7] take into $\mathrm{C}$ and the whitening matrix can be figured out as follow:

$G=D^{-1 / 2} E^{T}$

Where $D=\operatorname{diag}[\lambda(1), \cdots, \lambda(d)]$ and $\lambda(i)$ is the i-th biggest eigenvalue, $E=[c(1), \cdots, c(d)]$ and $c(i)$ is the eigenvector corresponding to $\lambda(i)$. After preprocessing, the mixed signal can be presented as follow:

$V_{d}=G X=M_{d} S+N^{\prime}$

where $M_{d}=G M$ is a $d \times d$ square matrix and $N^{\prime}=G N$ is a $d \times K$ noise matrix.

\subsection{Derivation of Extended Projection Algorithm}

By the rank-one decomposition, the feature vector $l_{1}$ of the one reply signal can be estimated in some $\Delta T$ period.
The unit orthogonal matrix $P=\left(l_{1} / \| l_{1}||, w_{2}, w_{3}, \cdots w_{d}\right)$ can be constructed, and it is $d \times d$ matrix, where $P \in C^{d}$. The $l_{1} /\left\|l_{1}\right\|$ is selected as the first vector and be used to constructed $w_{i}$, and $\forall i \in\{2, \cdots, d\}, w_{i}^{H} l_{1}=0$. Thereinto,

$\|\bullet\|$ stands for the Euclidean norm of a vector.

Assumption $P_{d-1}$ is the subset which constituted by the last $\mathrm{d}-1$ columns of $\mathrm{P}$ matrix. Then the resulting product $V_{d-1}=P_{d-1}^{H} V_{d}=P_{d-1}^{H} M_{d} S$. It is a $(d-1) \times K$ matrix. It contains only the rest of the other $\mathrm{d}-1$ signal information, such as

$V_{d-1}=P_{d-1}^{H} M_{d} S=P_{d-1}^{H} M_{d-1}\left(\begin{array}{l}s_{2} \\ \vdots \\ s_{d}\end{array}\right)=P_{d-1}^{H} M_{d-1} S_{d-1}$

Where $S_{d-1}$ are the last d-1 rows of S, and $M_{d-1}$ are the last $\mathrm{d}-1$ columns of $\mathrm{M}$. This equation indicates that a submatrix can be obtained, and the submatrix includes only d-1 signals from the mixed matrix including $\mathrm{d}$ signals.

It is easy to prove that $P_{d-1}^{H} M_{d-1}$ is a full rank square matrix. Then be $u_{1}$ the row produced by the maximum ratio combining beamformer, so :

$u_{1}=m_{1}^{H} V_{d}=\left\|m_{1}\right\|^{2} s_{1}+m_{1}^{H} M_{d-1} S_{d-1}$

Then the matrix $U$ can be described as follow:

$U=\left(\begin{array}{ll}V_{d-1}^{T} & u_{1}^{T}\end{array}\right)=\left(\begin{array}{lll}s_{2}^{T} & \cdots & s_{d}^{T}\end{array} \mid s_{1}^{T}\right)\left(\begin{array}{cc}\left(P_{d-1}^{H} M_{d-1}\right)^{T} & m_{1}^{H} M_{d-1} \\ 0 & \left\|m_{1}\right\|^{2}\end{array}\right)$

It's known that $\mathrm{S}$ is a $d \times K$ full rank matrix. Therefore, the matrix $U$ is full rank too. Performing Gram - Schmidt orthogonal decomposition to the matrix $U$, the mixed information can be isolated from $s_{2}, \cdots, s_{d}$. So the vector can be obtained which only contains $s_{1}$ information. That means that the $S_{1}$ has separated. Using the same method, the $S_{2}$ till $S_{d}$ can be separated one by one.

\subsection{Calculation Steps of the EPA}

The EPA algorithm can follows the next steps. 1) Slice matrix $X$ into numbers of segments, on which singular value decomposition (SVD) is performed. 2) For each slice, it need to decide if there are 0,1 or more sources. a) If there is at least one slice that contains only one source, go to step 3. b) If slices include either zero or more than one source, then selected the one which has the largest difference between the first and the second singular value. This choice is done in hope that the signature vector of the main source can be estimated. Go to step 3. c) If no source is detected, the algorithm stops. 3) Construct matrix in accordance with the 
Table 1. Information of source signals.

\begin{tabular}{|c|c|c|c|c|}
\hline Signal Formats & 1090ES ADS-B Signal 1 & 1090ES ADS-B Signal 2 & $\begin{array}{c}\text { A/C } \\
\text { Reply 1 }\end{array}$ & $\begin{array}{c}\text { A/C } \\
\text { Reply 2 }\end{array}$ \\
\hline \hline Beginning of signals $[\mu \mathrm{s}]$ & $t_{0}=0$ & $t_{1}=20$ & $t_{2}=40$ & $t_{3}=110$ \\
$t_{7}=140$ & $t_{6}=130.3$ \\
\hline Ending of signals $[\mu \mathrm{s}]$ & $t_{5}=120$ & $t_{7}$ & $t^{2}$ & \\
\hline
\end{tabular}

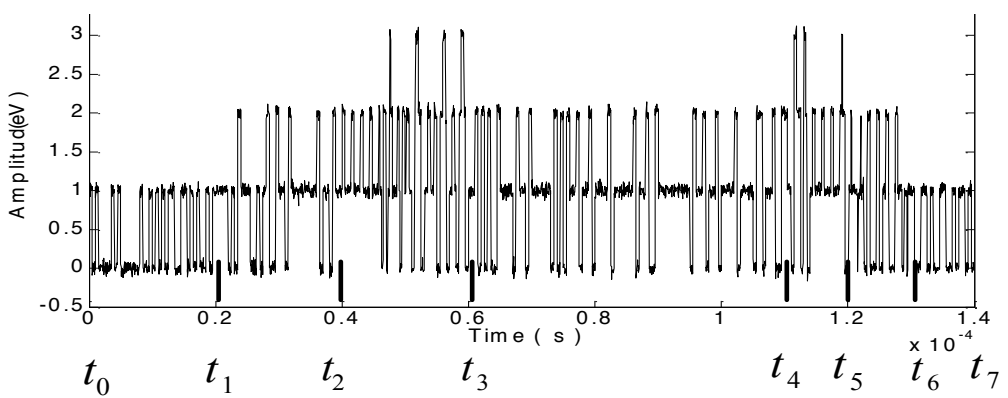

Fig. (1). Waveform of mixed signals.

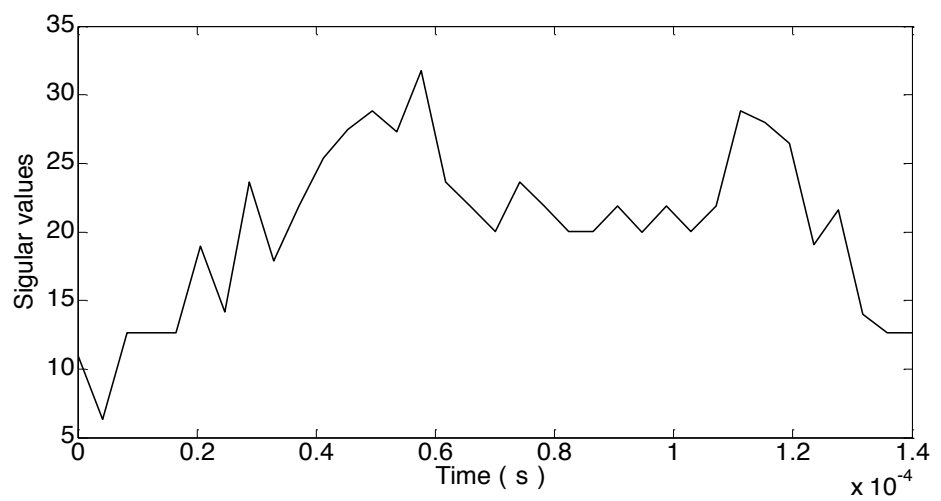

Fig. (2). Diagram of singular values and time after first SVD.

method described above, and the separated signals can be obtained one by one until the last signal is separated.

\section{SIMULATION AND PERFORMANCE ANALYSIS}

\subsection{Separation Signals Process and Analysis}

In the simulation, we use 1090ES and/or ADS-B coaxial antenna which has eight elements and is produced by SENSIS to receive four overlapped signals. The four signals include two 1090ES responsive signals and two A/C responsive signals. Specific information of the four signals is shown in Table 1. The waveforms of the overlapped signals are presented in Fig. (1). The received signals are sampled at the frequency of $f_{s}=20 \mathrm{MHz}$. At the same time, the sampled signals are whitened and dimension reduced. Then the data is sliced in segments of 80 samples $(4 \mu \mathrm{s})$, on which an SVD is performed.
According to the EPA calculation steps described in the above, the diagram of the singular values and time can be obtained after the first SVD decomposition, as shown in Fig. (2). Therefore, the first separated signal can be isolated, and shown in Fig. (3). With the same method, the diagram of the singular values and time is obtained after the second SVD as well as the other 1090ES responsive signal. They are respectively shown in Fig. (4), Fig. (5). In this paper, we don't discuss the two A/C separated signals obtained by EPA.

According to the mixed signals separation analysis results, the EPA method can effectively separate multiple $(d>3)$ overlapping signals. When the number of source signals is greater than 4 , it need only repeat steps described above until the last signal is separated. The separated results are shown in Fig. (6) and Fig. (7).

From the mixed signals separation analysis results, the sequence of the separated signals is unknown and that the amplitude of separated signals is different from that of 


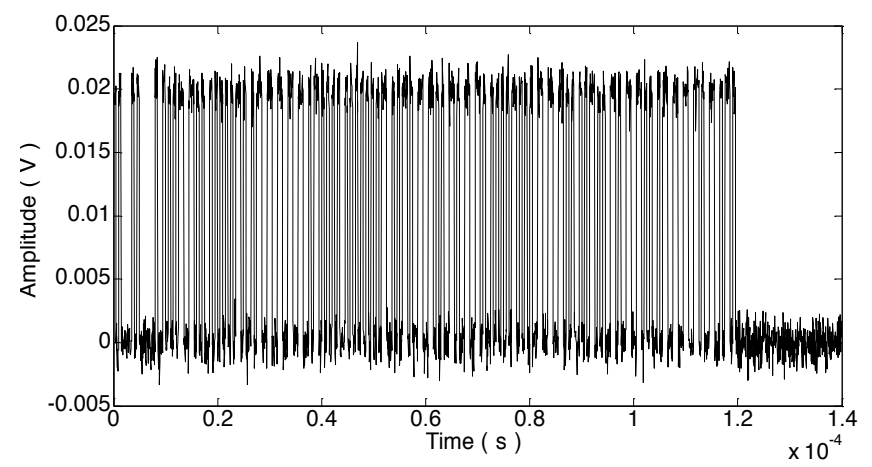

Fig. (3). Waveform of the first separated signal.

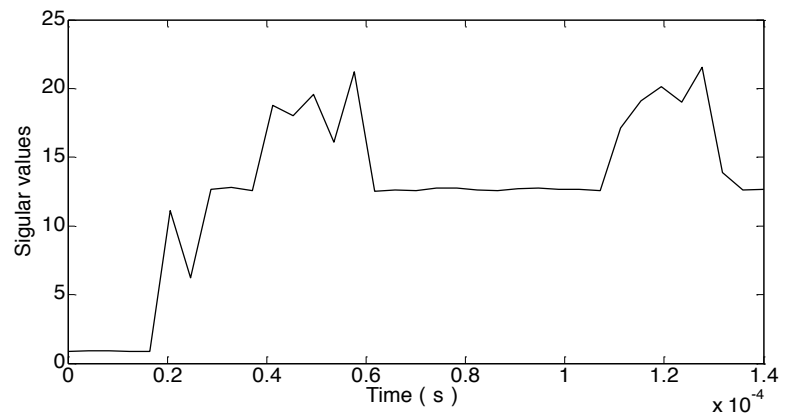

Fig. (4). Singular values versus time after the second SVD.

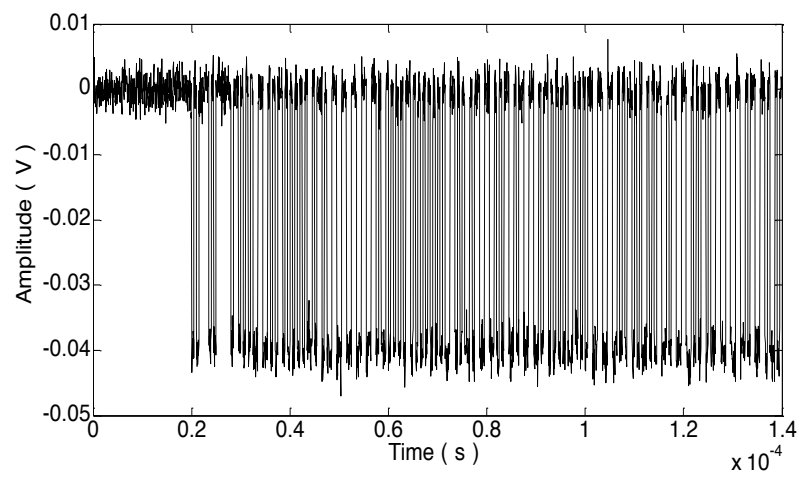

Fig. (5). Waveform of the second separated signal.

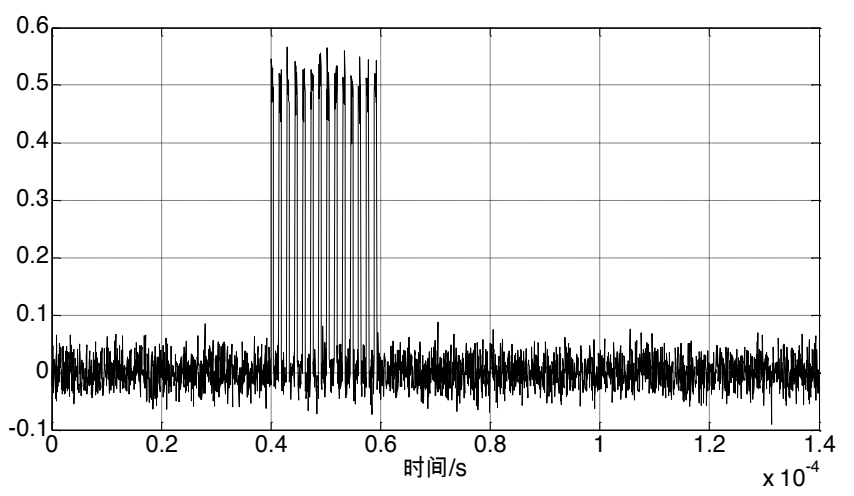

Fig. (6). Waveform of the third separated signal. 


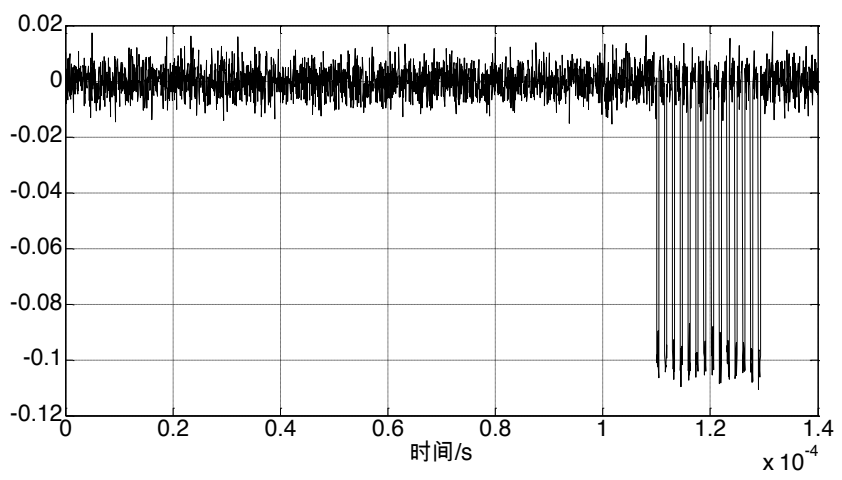

Fig. (7). Waveform of the Fourth separated signal.

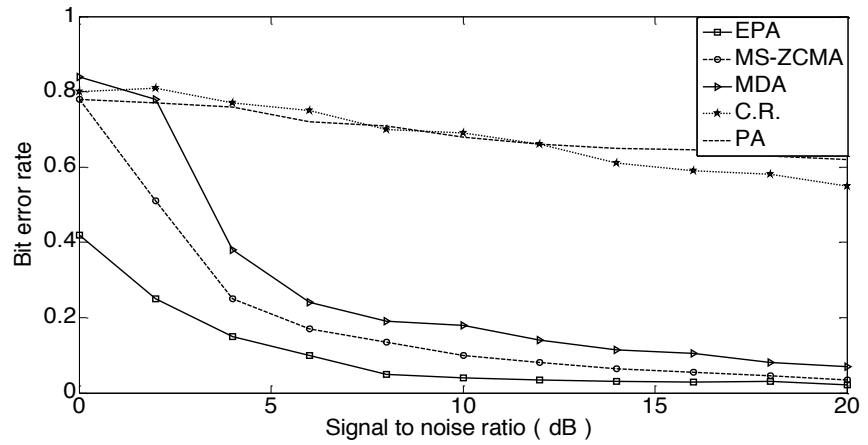

Fig. (8). Bit error rate versus SNR situations.

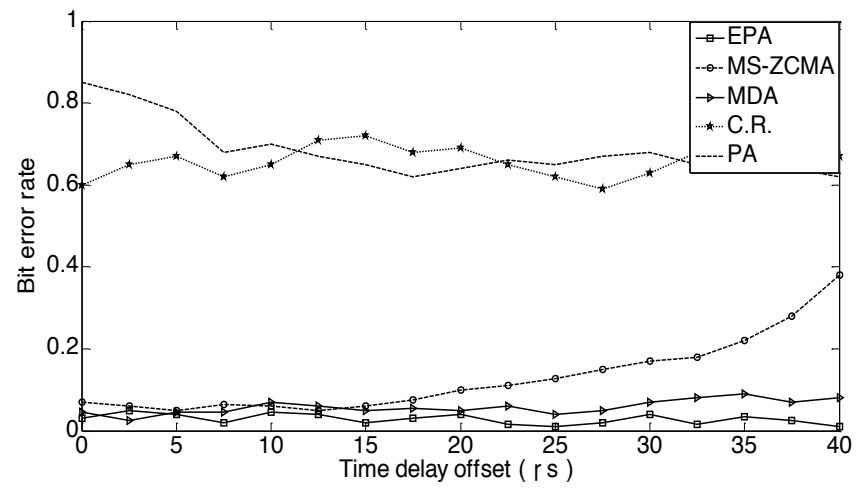

Fig. (9). Bit error rate versus time delay offset.

source signals. However, both signals sequences and signals amplitude do not have any impact on waveform. The unknown signals sequences make no difference in locating aircrafts because 1090ES signals carry the information of the identity of the aircraft itself.

Here, the stability of the EPA has been analyzed in details by verifying the impact on EPA made by signal to noise ratio (SNR) and time delay offset respectively. In the experiments, the 28000 sampling points are used and the 500 Monte-Carlo experiments are completed. In these experiments, it only need choose one 1090ES responsive signals separated by EPA to study the performance of the algorithm. At the same time, the performance of EPA, MDA and MSZCMA, PA and conventional receiver (CR) algorithm are analyzed and compared in details. And then the difference in the starting time of two 1090ES responsive signals as time delay offset is defined.
Firstly, the influence of the SNR on the algorithms is analyzed. When the SNR ranges from 0 to $20 \mathrm{~dB}$, and time delay offset is fixed to $6 \mu \mathrm{s}$. The bit error rate versus SNR is shown in Fig. (8). It clearly indicates that the bit error rate of the separated signal is very high in the case that SNR is lower than $5 \mathrm{~dB}$. The bit error rate decreases as the SNR increases. When the SNR is more than $12 \mathrm{~dB}$, bit error rate obtained by EPA is almost zero. It's also known that EPA performance is better than other algorithm in case of the same SNR.

In the Fig. (8), even if SNR reaches $20 \mathrm{~dB}$, the bit error rate of the signal obtained by the $\mathrm{CR}$ and PA is still very high. Because conventional receivers were not designed to receive two replies at a time, therefore it can not cope with more than one signal [7]. CR is presented here only for comparison with other algorithms. The result in Fig. (9) is obtained by the first signal only. In the literature [2], the PA is only applicable to the separation of the two overlapped sig- 


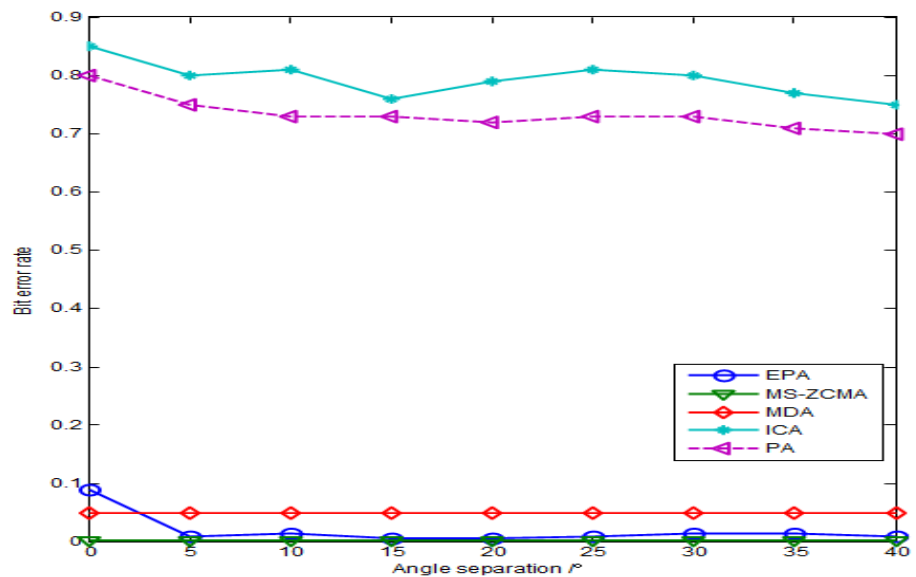

Fig. (10). Relationship between signal direction interval and the error rate.

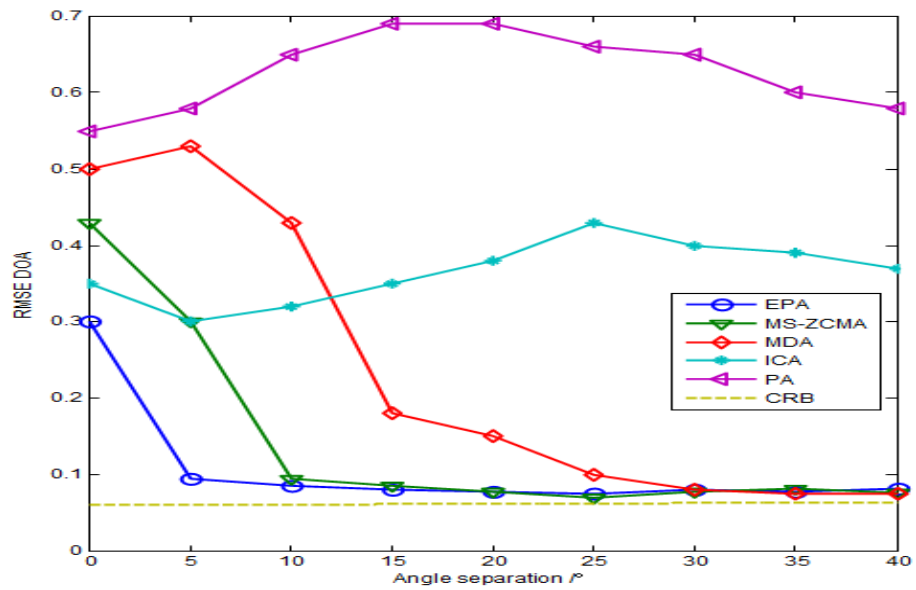

Fig. (11). Relationship between signal direction interval and the root mean square error of DOA.

nals. When the number of signal sources is greater than two, PA is no longer applicable. Therefore the PA is presented here for the need for comparative analysis as well.

Secondly, the time delay offset is analyzed. The fixed SNR of each source is equal to $14 \mathrm{~dB}$, and the time delay offset ranges from 0 to $40 \mu \mathrm{s}$. The bit error rate versus time delay offset is shown in Fig. (9). In the Fig. (9), it clearly appears that the bit error rate of the separated signal obtained by both EPA and MS-ZCMA is almost not influenced by the time delay offset. The figure indicates that the performance of EPA is better than that of MS-ZCMA in case of the same time delay offset. However, MS-ZCMA is very complex.

In the Table 2, it is shown that computational complexity of MS-ZCMA is higher than that of EPA. The complex computation of MS-ZCMA will increase hardware requirements, or it would be difficult to ensure that the 1090ES responsive signals could get real-time processing. In the literature [4], a sharp rise of the bit error rate of the separated signals obtained by MDA appears when the time offset is greater than $40 \mu \mathrm{s}$, which means that MDA is not reliable when the time delay offset is above $40 \mu \mathrm{s}$. As mentioned above, both CR and PA can't be used for the separation of multiple signals. The two algorithms comparative analyses are presented in here.

Table 2. Computational complexity of EPA and MS-ZCMA.

\begin{tabular}{|c|c|c|}
\hline Separation Algorithm & EPA & MS-ZCMA \\
\hline \hline Computational complexity & $\mathrm{O}\left(\mathrm{K} \times \mathrm{d}^{3}\right)$ & $\mathrm{O}\left(\mathrm{K} \times \mathrm{d}^{8}\right)$ \\
\hline Computational time $[\mathrm{s}]$ & 0.056 & 0.47 \\
\hline
\end{tabular}

From the Fig. (10), the results can be presented. When the signal direction interval is less than 6 degrees, the PA algorithm and ICA algorithm is going to collapse firstly. The source signal with small direction interval can be separated only with EPA, MS-ZCMA algorithm.

From the Fig. (11), the results can be presented. With the increase of the signal direction interval, the results of the EPA, MS-ZCMA algorithm can infinitely approach the Cramer-Rao Bound (CRB). When the signal direction interval is smaller, the performances of the all algorithms have some improvements. 


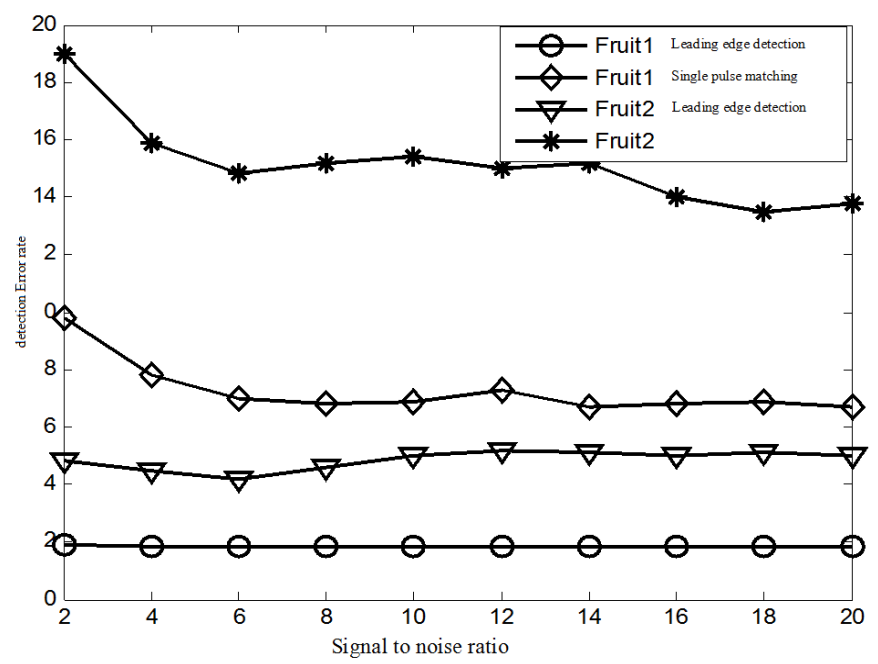

Fig. (12). Detection error rate of preamble.

\subsection{Detection of the Preamble}

In the no A/C fruit situation, 1090ES signals' preamble the autocorrelation detection method can detect the preamble and its TOA. When the threshold selects as $28 \mathrm{~V}$ (without normalization), the detection point quantity which is bigger than threshold, is more than 7 consecutive points, so it is considered a effective peak.

In the actual situation, the 1090ES signals can be interfered by the $\mathrm{A} / \mathrm{C}$ fruit. In the $\mathrm{A} / \mathrm{C}$ fruit situation, the preamble the autocorrelation detection method is failure completely. At this time, in the case of choice of the same threshold $(28 \mathrm{~V})$, the real wave peak can not be correctly identified.

Because of the 1090ES and ADS-B signal leading pulse autocorrelation detection method can not be used when the signals are interfered by $\mathrm{A} / \mathrm{C}$ fruit, the single pulse correlation detection method is presented in paper. That is to say the correlation is used for single pulse.

Only a single pulse can be used to match filter with detected signal, the single pulse width is $0.5 \mu \mathrm{s}$. And then the position of every pulse is being located.

Firstly, the correlation calculation is operation between the reference pulse (It is single pulse) and the detected signal, the waveform of the output result of the correlation operation is a triangle. Then a certain threshold can be set, the threshold can be used as criterion for upper and lower boundary of the peak of each sub pulse. The maximum value of the upper and lower boundary can be considered as the pulse position. In order to eliminate the influence of the isolated noise points, the width limitation can be set for those beyond the threshold points. If less than 6 consecutive points(the 6 continuous points situation is an at the sampling rate of $20 \mathrm{MHz}$ ), the isolated noise points can be abandoned.

Suppose, the signals sampling frequency is $20 \mathrm{MHz}$, the rising edge of the pulse is $30-50 \mathrm{~ns}$, signal to noise ratio is $10 \mathrm{~dB}$, the SSR A/C mode signal interference is 40,000 times/s, each $\mathrm{A} / \mathrm{C}$ mode signal is independent of each other, and uniform distribution in a certain time(such as $1 \mathrm{~s})$. The analysis results are presented in Fig. (12).

Fruit 1-One A/C mode interference is fixed superimposed on 1090ES preamble.

Fruit 2-Two A/C mode interference is fixed superimposed on 1090ES preamble.

And then we can calculated the superimposed probability on 1090ES signals interferences, there are 2-12 superimposed interferences on 112 bits of the 1090ES signal. The superimposed probability is $2.52 \%, 6.15 \%, 10.96 \%, 15.20 \%$, $17.07 \%, 15.97 \%, 12.69 \%, 8.69 \%, 5.19 \%, 2.73 \%$, and $1.27 \%$. The total is $98.44 \%$.

As presented in the Fig. (12), the correct rate of the leading pulse edge detection method is better than the single pulse matching detection method slightly. And the accuracy rate of existing one $\mathrm{A} / \mathrm{C}$ mode interference is higher than existing two A/C mode interference. At same time, the influence come from signal to noise ratio changing is not clear between the two detection methods.

\section{CONCLUSION}

In this paper, we have analyzed the performances of the location signals separation algorithms and then presented the application of EPA to separate 1090ES and/or ADS-B signals. The results indicated that EPA can separate many responsive signals one by one. The algorithm makes use of matrix reorganization and SVD to get the estimated signal. The algorithm has the following advantages:

1) The algorithm can successfully separate 1090ES and/or ADS-B signals from multiple $(\mathrm{d}>3)$ overlapped signals.

2) The algorithm has low computational complexity. 
3) The algorithm is unaffected by the time delay offset between two 1090ES and/or ADS-B signals.

Experimental simulation and analysis presents that the EPA algorithm is not only simple but also highly stable when it is applied to separate 1090ES and/or ADS-B signals.

\section{CONFLICT OF INTEREST}

The authors confirm that this article content has no conflicts of interest.

\section{ACKNOWLEDGEMENTS}

This work is financially supported by the National Natural Science Foundation of China (No. U1233112 and No. 61079008), and then supported by the research plan foundation of the application foundation and advanced techniques of Tianjin (Key projects) (No. 11JCZDJC25200). At same time, it supported by Central University Fund Project of Ministry of Education (No. 3122014P001), too.

\section{REFERENCES}

[1] Lin Xi, Zhang Jun, Zhu Yanbo, et al. "Surveillance accuracy analysis of ADS-B supporting the separation service in western China". Integrated Communications, Navigation and Surveillance Conference, 2009. ICNS '09. Valencia, Spain. 13-15 May 2009: 1-6.

[2] N. Petrochilos, G. Galati and E. Piracci. "Projection Techniques for Separation of Multiple Secondary Surveillance Radar Sources in a Real Environment", Proc. of IEEE SAM 2006 (Waltham, July 1214, 2006). p. 344-353.

[3] M. Zhou and A.J. van der Veen. "Computational Advances in Multi-Sensor Adaptive Processing". 2011 4th IEEE International Workshop on CD-ROM (December 13-16, 2011). P.181-184.
[4] Petrochilos, N., van der Veen, A.J. "Algorithms to separate overlapping secondary surveillance radar replies". IEEE International Conference on Acoustics, Speech, and Signal Processing, 2004. (ICASSP '04). Montreal, Quebec, Canada. 17-21 May 2004, 2(2): 49-52.

[5] Petrochilos, N., van der Veen, A.J. “Algebraic Algorithms to Separate Overlapping Secondary Surveillance Radar Replies". IEEE Transactions on Signal Processing. July 2007, 55(7): 3746-3759.

[6] Petrochilos, N., Galati, G., Piracci, E. "Separation of SSR Signals by Array Processing in Multilateration Systems". IEEE Transactions on Aerospace and Electronic Systems. July 2009, 45(3): 965-982.

[7] Hefa Zhang. "Research on blind signal Separation and Its Application in Passive Location", University of Electronic Science and Technology of China, 2011. (In Chinese).

[8] Petrochilos. N, Piracci, E.G., Galati, G. "Improved MDA, a case for de-garbling SSR mode S replies". 2014 Tyrrhenian International Workshop on Digital Communications - Enhanced Surveillance of Aircraft and Vehicles, TIWDC/ESAV 2014, p 87-91, November 3 , 2014.

[9] G. Galati, M. Leonardi, E.G. Piracci, N. Petrochilos, S. Samanta, "The transponder Data Recorder: Implementation and First Results", IEEE Aerospace and Electronic Systems magazine, February 2014, 29(2): 6-13.

[10] Marchi, Pablo G., Galarza, Cecilia G. "Techniques to Separate Multiple Responses in Secondary Surveillance Radars Operated in $S$ Mode". 2014 IEEE Biennial Congress of Argentina, ARGENCON 2014, p 245-250.

[11] Satapathy, G., Chen, J., Tolani, D., Sturdy, J.L., Henion, J.T., Kubat, G. "A Traffic Information Service-Broadcast Model for Mixedequipage Aircraft Simulation". 2010 Integrated Communications Navigation and Surveillance Conference (ICNS), 2010 ICNS: E3-1 - E3-15.

[12] Leonardi, M., Piracci, E., Galati, G. “ADS-B Vulnerability to Low Cost Jammers: Risk assessment and Possible Solutions Digital Communications". Enhanced Surveillance of Aircraft and Vehicles (TIWDC/ESAV), 2014 Tyrrhenian International Workshop on, TIWDC-ESAV 2014: 41-46.

(C) Fengxun et al.; Licensee Bentham Open.

This is an open access article licensed under the terms of the Creative Commons Attribution Non-Commercial License (http://creativecommons.org/licenses/by-nc/3.0/) which permits unrestricted, non-commercial use, distribution and reproduction in any medium, provided the work is properly cited. 